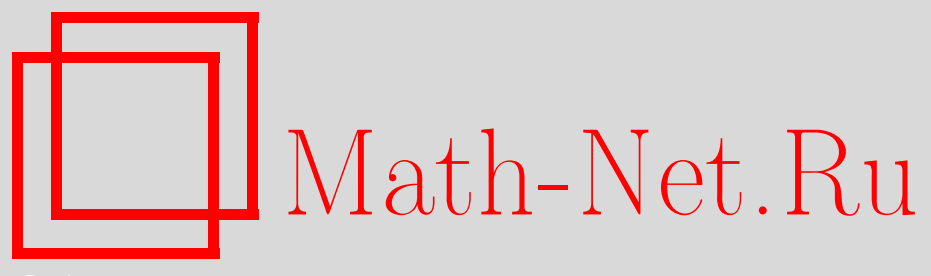

А. В. Покровский, Устранимые особенности решений нелинейных эллиптических уравнений, УМH, 2007, том 62, выпуск 3, 215-216

DOI: https://doi.org/10.4213/rm6423

Использование Общероссийского математического портала Math-Net.Ru подразумевает, что вы прочитали и согласны с пользовательским соглашением http://www . mathnet.ru/rus/agreement

Параметры загрузки:

IP : 54.80 .73 .141

26 апреля 2023 г., $17: 41: 11$

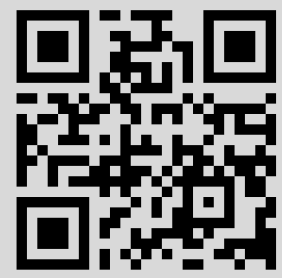




\section{Устранимые особенности решений нелинейных эллиптических уравнений}

\section{А. В. Покровский}

Пусть $G$ - ограниченная область в $\mathbb{R}^{n}(n \geqslant 2)$, и пусть

$$
A(u)=\sum_{i, j=1}^{n} D_{i}\left(a_{i j}(x, u, \nabla u) D_{j} u\right)+\sum_{i=1}^{n} b_{i}(x, u, \nabla u) D_{i} u+c(x, u, \nabla u) u
$$

- нелинейный дифференциальный оператор, удовлетворяющий следующему условию эллиптичности в $G$ : для каждого ограниченного множества $K \subset \mathbb{R}$ существует такое $\lambda_{K}>0$, что неравенство $\sum_{i, j=1}^{n} a_{i j}(x, z, p) q_{i} q_{j} \geqslant \lambda_{K}|q|^{2}$ выполняется при всех $x \in G, z \in K, p \in K^{n}$ и $q=\left(q_{1}, \ldots, q_{n}\right) \in \mathbb{R}^{n}$. Здесь функции $a_{i j}(x, z, p), b_{i}(x, z, p)$ и $c(x, z, p)$ (в настоящей работе мы рассматриваем только измеримые вещественные функции) определены на множестве $G \times \mathbb{R} \times \mathbb{R}^{n}(i, j=\overline{1, n}), \nabla u=\left(D_{1} u, \ldots, D_{n} u\right)=$ $\left(\frac{\partial u}{\partial x_{1}}, \ldots, \frac{\partial u}{\partial x_{n}}\right),|q|=\left(q_{1}^{2}+\cdots+q_{n}^{2}\right)^{1 / 2}$. Мы также предполагаем, что для каждого множества $K \Subset \mathbb{R}$ существует такое $M_{K}>0$, что, каковы бы ни были $x, y \in G$, $z, w \in K$ и $p, q \in K^{n}$, имеет место неравенство

$$
\begin{aligned}
& \sum_{i, j=1}^{n}\left|a_{i j}(x, z, p)-a_{i j}(y, w, q)\right|+\sum_{i=1}^{n}\left|b_{i}(x, z, p)-b_{i}(y, w, q)\right| \\
& \quad+|c(x, z, p)-c(y, w, q)| \leqslant M_{K}\left(|x-y|^{\gamma}+|z-w|^{\gamma}+|p-q|\right),
\end{aligned}
$$

где $\gamma \in(0,1]$ зависит только от оператора $A$. В дальнейшем если хотя бы одна из функций $a_{i j}(x, z, p), b_{i}(x, z, p)$ и $c(x, z, p)(i, j=\overline{1, n})$ зависит от $x$, то мы считаем, что граница области $G$ принадлежит классу $C^{1, \gamma}$. Предположим, что $c(x, z, p) \leqslant 0$ всюду в $G \times \mathbb{R} \times \mathbb{R}^{n}$.

Пусть $E$ - непустое замкнутое множество в $G, E \neq G, \alpha \in(0,1)$. Как обычно, $C^{1, \alpha}(G)$ - линейное пространство всех непрерывно дифференцируемых функций в $G$, для которых конечна норма

$$
\left\|f\left|C^{1, \alpha}(G) \|:=\sup _{G}\right| f \mid+\sum_{i=1}^{n}\left(\sup _{G}\left|D_{i} f\right|+\sup _{x, y \in G, x \neq y} \frac{\left|D_{i} f(x)-D_{i} f(y)\right|}{|x-y|^{\alpha}}\right) .\right.
$$

Множество $E$ назовем $A$-устранимым в классе $C^{1, \alpha}(G)$, если любая функция $u \in$ $C^{1, \alpha}(G)$, для которой при всех $\varphi \in C_{0}^{\infty}(G \backslash E)$ выполняется равенство

$$
\int_{G}\left(\sum_{i, j=1}^{n} a_{i j}(x, u, \nabla u) D_{j} u D_{i} \varphi-\sum_{i=1}^{n} b_{i}(x, u, \nabla u) D_{i} u \varphi-c(x, u, \nabla u) u \varphi\right) d x=0
$$

может быть так переопределена на $E$ с сохранением ее принадлежности к $C^{1, \alpha}(G)$, что после этого переопределения последнее равенство будет иметь место при всех $\varphi \in C_{0}^{\infty}(G)$.

При сделанных выше предположениях и во введенных обозначениях имеет место следующая теорема.

Tеорема 1. Пусть $0<\alpha \leqslant \gamma \leqslant 1 \quad u \quad \alpha<1$. Тогда для А-устранимости множества $E$ в классе $C^{1, \alpha}(G)$ необходимо и достаточно равенство нулю его хаусдорфовой мери mes $^{n-1+\alpha} E$ порядка $n-1+\alpha$. 
Для уравнения Лапласа $\Delta u=0$ теорема 1 установлена в работах [1], [2], для уравнения минимальных поверхностей $\operatorname{div}\left(\frac{\nabla u}{\left(1+|\nabla u|^{2}\right)^{1 / 2}}\right)=0-$ в [3] (с $\left.\gamma=1\right)$. Доказательство в общем случае проводится с помощью рассуждений из последней работы. Поэтому мы остановимся только на его основных моментах.

Достаточность условия $\operatorname{mes}^{n-1+\alpha} E=0$ проверяется с использованием леммы о разбиении единицы из работы [4]. Установим его необходимость. Пусть $\mathrm{mes}^{n-1+\alpha} E>0$. Выделяя подмножество, можно без уменьшения общности считать, что $E$ - компакт в $G$ без внутренних точек. По лемме Фростмана (см. [5]) существует такая борелевская мера $\mu$ с носителем на $E$, что $\mu(E)>0$ и $\mu(B(x, r)) \leqslant r^{n-1+\alpha}$ для любого шара $B(x, r):=\left\{y \in \mathbb{R}^{n}:|y-x|<r\right\}(r>0)$. Пусть $t>0, f \in C^{1, \alpha}(G)$, и пусть $g_{t}$ - функция из соболевского класса $W_{0}^{1,2}(G)$, являющаяся обобщенным решением уравнения

$$
\sum_{i, j=1}^{n} D_{i}\left(a_{i j}(x, f, \nabla f) D_{j} g_{t}\right)+\sum_{i=1}^{n} b_{i}(x, f, \nabla f) D_{i} g_{t}+c(x, f, \nabla f) g_{t}=-t \mu .
$$

Переписывая правую часть этого уравнения в виде $t \operatorname{div}(\nabla h)$, где функция $h \in W_{0}^{1,2}(G) \cap C^{1, \alpha}(G)$ является обобщенным решением уравнения $\Delta u=-\mu$ (см. [3]), и пользуясь известными результатами из теории линейных равномерно эллиптических уравнений второго порядка в дивергентной форме [6; теоремы 8.3, 8.16, 8.33 и 8.34], мы заключаем, что функция $g_{t}$ существует, единственна и принадлежит классу $C^{1, \alpha}(G)$, а отображение $F_{t}: f \rightarrow g_{t}$ непрерывно по норме пространства $C^{1}(G)$ и при малых $t$ переводит в себя единичный шар пространства $C^{1, \alpha}(G)$. По теореме Шаудера (см. [6; теорема 11.1]) отсюда следует, что при достаточно малом $t$ это отображение имеет неподвижную точку $u_{t}$. Это означает, что функция $u_{t}$ принадлежит классу $C^{1, \alpha}(G)$ и является обобщенным решением уравнения $A\left(u_{t}\right)=-t \mu$. Поэтому множество $E$ не является $A$-устранимым в классе $C^{1, \alpha}(G)$. Если функции $a_{i j}(x, z, p)$, $b_{i}(x, z, p)$ и $c(x, z, p)(i, j=\overline{1, n})$ не зависят от $x$, то в приведенных рассуждениях можно заменить область $G$ на любой содержащий ее открытый шар, и условие на гладкость границы в этом случае излишне. Теорема 1 доказана.

Теорема 1 применима к линейным равномерно эллиптическим уравнениям в дивергентной форме с гёльдеровыми коэффициентами, к уравнению капиллярности $\operatorname{div}\left(\frac{\nabla u}{\left(1+|\nabla u|^{2}\right)^{1 / 2}}\right)+c u=0(c=$ const $\leqslant 0)$ с $\gamma=1$, к уравнению Эмдена-Фаулера $\Delta u-|u|^{p-1} u=0(p>1)$ с $\gamma=\min \{1, p-1\}$ и к уравнению $\Delta u-u|\nabla u|^{2}=0$ с $\gamma=1$.

\section{Список литературы}

[1] Е. П. Долженко, Изв. АН СССР. Сер. матем., 28:5 (1964), 1113-1130. [2] Е. П. Долженко, Изв. АН СССР. Сер. матем., 28:6 (1964), 1251-1270. [3] А. В. Покровский, Функи. анализ и его прил., 39:4 (2005), 62-68. [4] R. Harvey, J. Polking, Acta Math., 125:1/2 (1970), 39-56. [5] Л. Карлесон, Избранные проблемы теории исключительных множеств, Мир, М., 1971. [6] Д. Гилбарг, Н. Трудингер, Эллиптические дифференциалъные уравнения с частными производными второго порядка, Наука, М., 1989.

А. В. Покровский (А. V. Pokrovskii)

Институт математики НАН Украины

E-mail: pokrovsk@imath.kiev.ua
Представлено Л. Р. Волевичем Принято редколлегией 08.02.2007 